\title{
A genome-wide study of PDZ-domain interactions in C. elegans reveals a high frequency of non-canonical binding
}

\author{
Nicolas Lenfant ${ }^{1 \dagger}$, Jolanta Polanowska ${ }^{1,3 \dagger}$, Sophie Bamps ${ }^{1,2}$, Shizue Omi ${ }^{1,3}$, Jean-Paul Borg ${ }^{1}$, Jérôme Reboul ${ }^{1,3^{*}}$
}

\begin{abstract}
Background: Proteins may evolve through the recruitment and modification of discrete domains, and in many cases, protein action can be dissected at the domain level. PDZ domains are found in many important structural and signaling complexes, and are generally thought to interact with their protein partners through a C-terminal consensus sequence. We undertook a comprehensive search for protein partners of all individual PDZ domains in C. elegans to characterize their function and mode of interaction.

Results: Coupling high-throughput yeast two-hybrid screens with extensive validation by co-affinity purification, we defined a domain-orientated interactome map. This integrates PDZ domain proteins in numerous cell-signaling pathways and shows that PDZ domain proteins are implicated in an unexpectedly wide range of cellular processes. Importantly, we uncovered a high frequency of non-canonical interactions, not involving the C-terminus of the protein partner, which were directly confirmed in most cases. We completed our study with the generation of a yeast array representing the entire set of PDZ domains from C. elegans and provide a proof-of-principle for its application to the discovery of PDZ domain targets for any protein or peptide of interest.

Conclusions: We provide an extensive domain-centered dataset, together with a clone resource, that will help future functional study of PDZ domains. Through this unbiased approach, we revealed frequent non-canonical interactions between PDZ domains and their protein partners that will require a re-evaluation of this domain's molecular function.

[The protein interactions from this publication have been submitted to the IMEx (http://www.imexconsortium.org) consortium through IntAct (PMID: 19850723) and assigned the identifier IM-14654]
\end{abstract}

\section{Background}

Because of its biological importance, the PDZ (PSD-95, Discs-large, ZO-1) domain has been intensively studied at the structural and functional level. Proteins containing PDZ domains frequently serve as molecular scaffolds, which assemble signaling complexes needed for efficient and specific signal transduction at defined subcellular sites, such as at polarized epithelial cell junctions, or synapses in neurons [1-3]. Early work indicated a preferential interaction between PDZ domains and the C-terminal amino acids of target proteins [4]. In some

\footnotetext{
* Correspondence: jerome.reboul@inserm.fr

† Contributed equally

'Inserm, U891, CRCM, Marseille, F-13009, France; Institut Paoli-Calmettes, Marseille, F-13009, France; Univ Méditerranée, F-13007, Marseille, France Full list of author information is available at the end of the article
}

cases, removal of the $3 \mathrm{C}$-terminal residues of the partner protein abrogates interaction with the PDZ domain [5]. Much subsequent effort has been put into bioinformatic studies and small- and large-scale screens to refine the exact sequence of this presumed C-terminal motif [5-10], leading to several consensus sequences, with different degrees of refinement (e.g. Additional file $1 ;[1,10-12])$. Individual proteins can contain multiple PDZ domains. For example, the human multiple PDZ domain protein (MPDZ) has 13. When their interactions with other proteins have been dissected, the different PDZ domains of a single protein often have been found to have distinct binding partners (see for example the Uniprot entry for MPDZ [13]). PDZ domain proteins have also been used in the context of large-scale searches for protein partners. For example, global
C Biomed Central

(c) 2010 Lenfant et al; licensee BioMed Central Ltd. This is an Open Access article distributed under the terms of the Creative Commons Attribution License (http://creativecommons.org/licenses/by/2.0), which permits unrestricted use, distribution, and reproduction in any medium, provided the original work is properly cited. 
interactome studies with $C$. elegans proteins assayed the interactions of 25 of the nematode's 62 PDZ domain proteins. Although these 25 proteins were found to be involved in 218 interactions, whether the different PDZ domains played a direct role was not addressed [14,15]. No comprehensive, proteome-wide screen using all PDZ domains, however, has been reported for any organism.

Here, we describe the characterization and cloning of every single one of the $93 \mathrm{PDZ}$ domains from C. elegans. We generated a versatile resource, with each domain in the Gateway system, allowing facile transfer to different expression systems. As an example, we made a yeast array of the 93 PDZ domains and provide a proof-of-principle for its application to the discovery of PDZ domain targets for any protein or peptide of interest. In addition, from a separate yeast two-hybrid $(\mathrm{Y} 2 \mathrm{H})$ screen, we identified more than 650 potential partners for these domains. A large number of these interactions were independently validated using a coimmunoprecipitation approach. An analysis of these interactors implicates PDZ domains in a broad range of cellular functions. Unexpectedly, many of the interactions did not involve a C-terminal consensus sequence, suggesting that PDZ domains frequently bind their partners in a hitherto uncharacterized mode.

\section{Results}

\section{An interactome map for PDZ domains}

We chose to define the interaction partners of all the PDZ-domain proteins in C. elegans. Through an exhaustive cross-database search, we identified a total of 93 PDZ domains in 62 distinct proteins, not counting isoforms sharing domains (see Additional file 2: Supplemental Table S1). Among these PDZ-domain containing proteins, only $44 \%$ were associated with any gene ontology annotation based on experimental data ([16]; Additional file 2: Supplemental Table S2). The DNA for all 93 domains was amplified and cloned. The insert for each clone was sequenced-verified, and this comprehensive clonal collection, in the Gateway entry vector allowing rapid transfer into multiple other vectors $[17,18]$, is available as a community resource upon request. The inserts were all transferred into a DBvector and used in high-stringency $\mathrm{Y} 2 \mathrm{H}$ screens against the non-normalized cDNA library AD-wrmcDNA [18]. We pulled out 447 interactions involving 317 interacting proteins and 75 individual PDZ domains. 6 PDZ domains were auto-activators and therefore not included in the screen, thus $81 \%$ of the PDZ domains gave at least 1 interaction, with a mean of 6 interacting proteins (see Additional file 3: Supplemental Tables S3, S4 and S5). As expected, there was limited overlap with the results of the previous global $C$. elegans $\mathrm{Y} 2 \mathrm{H}$ screens due in part to the incomplete and disparate degrees of coverage (6 shared interactions with Worm Interactome 8 [15]), and the domain-nature of the current screen. We did observe a clear bias towards proteins containing C-terminal class I consensus motifs (as defined in Additional file 1). One striking observation, however, was the high frequency (51\%) of interacting proteins that did not possess a classical C-terminal consensus sequence (Table 1; Additional file 3: Supplemental Table S6). This trend was maintained even when interacting proteins that had multiple PDZ-domain partners were counted only once in the analysis (Table 1). This opened the possibility to perform a second screen using the ADORFeome library [19], which has the advantage of being highly normalized. In this library, the stop codon of each insert is replaced by the $\mathrm{B} 2$ recombination sequence, giving rise to proteins with a constant nonnative $\mathrm{C}$-terminal extension. These additional 8 amino acids (PAFLYKVV) do not correspond to the consensus binding sequence for native PDZ-domains. Using this library, we identified a total of 227 interactions involving 178 interacting proteins and 59 PDZ domains (see Additional file 3: Supplemental Table S3). These included 14 in common with the cDNA screen. This degree of overlap $(6 \%)$ is slightly lower than that reported [14,19] for previous screens against the two libraries (14\% and $16 \%$, respectively), possibly reflecting the fact that our PDZ

Table 1 Proportions of C-terminal consensus classes in interacting proteins

\begin{tabular}{|c|c|c|c|c|c|c|}
\hline & consensus class 1 & consensus class 2 & consensus class 3 & total consensus & total non consensus & \\
\hline AD-wrmcDNA library & $20 \%$ & $24 \%$ & $5 \%$ & $49 \%$ & $51 \%$ & $n=447$ \\
\hline AD-ORFeome library & $10 \%$ & $18 \%$ & $5 \%$ & $33 \%$ & $66 \%$ & $n=227$ \\
\hline \multirow[t]{2}{*}{ C. elegans proteome } & $8 \%$ & $18 \%$ & $5 \%$ & $31 \%$ & $69 \%$ & $n=20186$ \\
\hline & consensus class 1 & consensus class 2 & consensus class 3 & total consensus & total non consensus & \\
\hline AD-wrmcDNA library & $18 \%$ & $21 \%$ & $5 \%$ & $44 \%$ & $56 \%$ & $\mathrm{n}=317 \mathrm{nr}$ \\
\hline AD-ORFeome library & $9 \%$ & $15 \%$ & $4 \%$ & $28 \%$ & $72 \%$ & $\mathrm{n}=178 \mathrm{nr}$ \\
\hline C. elegans proteome & $8 \%$ & $18 \%$ & $5 \%$ & $31 \%$ & $69 \%$ & $n=20186$ \\
\hline
\end{tabular}

Proportions of C-terminal consensus classes for interacting proteins identified in AD-wrmcDNA and AD-ORFeome screens compared with proportions of Cterminal consensus classes in the complete C. elegans proteome (WS190 [16]) are given. Consensus class 1: [ST]X[FWCYMVILA], Consensus class 2 [YFWCMVILA]X [YFWCMVILA], Consensus class 3 [DE]X[YFWCMVILA] (X: any amino acid). Upper panel: proportions calculated using all PDZ-interacting protein pairs, lower panel: proportions calculated using each interacting protein only once when interacting with multiple PDZ domains (nr stands for non-redundant). 
domain screen is biased towards native $\mathrm{C}$-terminus consensus motifs, which are not accessible in the AD-ORFeome library. Within these 59 PDZ domains (63\% of the total; mean 3.8 interacting proteins per domain; see Additional file 3: Supplemental Tables S4 and S5), the prevalence of $\mathrm{C}$-terminal consensus sequences in the interacting proteins reflected that seen in the proteome as a whole (Table 1; Additional file 3: Supplemental Table S6). Taken together, these results suggest that some PDZ domains might interact with their target ligands outside the $\mathrm{C}$-terminus much more often than expected.

\section{Characteristics of PDZ domain interacting proteins}

Pooling the results from the two screens, we obtained 674 interactions involving 469 proteins and 78 PDZ domains (from 55 proteins out of the original 62)(Figure 1A). In many cases, single proteins were found to interact with multiple PDZ domains (Figure 1B), consistent with the known promiscuity of ligand-PDZ domain interactions [20]. Gene Ontologies (GO) [21] analysis are limited by the fact that only $182 / 469$ proteins have attributes inferred from experimental evidence. We therefore opted to extend this analysis with a manual curation of our protein set based principally on Wormbase [16] annotations (Figure 2, Additional file 4: Supplemental Tables S7, S8 and S9). PDZ domains have long been known to be involved in the scaffolding of proteins complexes at the plasma membrane thus contributing to the signaling specificity of many receptors, notably at the synapse [22], or to the establishment and maintenance of epithelial polarity [2,23]. Indeed, just under half of the functionally annotated proteins are involved in signaling (protein kinases, GTPases and phosphatases), structural maintenance or transport. The annotation of the other PDZ interacting proteins reveals a broad range of functions ranging from metabolism, ubiquitination, RNA binding and processing to transcriptional regulation (Figure 2). Interestingly some studies indicate nuclear roles for PDZ domain proteins. For example, the junctional protein $\mathrm{ZO}-2$ directly interacts in the nucleus with the DNA-binding protein scaffold attachment factor-B (SAF-B) [24].

\section{Frequent use of non-consensus binding confirmed by co-IP}

It is well established that some $\mathrm{Y} 2 \mathrm{H}$ interactions do not reflect a physiologically relevant binding events between proteins. Ideally, these interactions need to be validated in vivo. Such tests are fastidious and not compatible with large-scale studies. We therefore sought to assay a subset of our $\mathrm{Y} 2 \mathrm{H}$ interactions, using a very distinct experimental system, namely co-affinity immunoprecipitations (co-IP) from human $293 \mathrm{~T}$ cells. First, we choose
13 different interactions, found in the cDNA screen, involving $11 \mathrm{PDZ}$ domains and 9 interacting proteins. All these interactions were detected by co-IP using constructs encompassing the PDZ interacting full-length proteins, with their native C-terminus (Figure 3, Additional file 5: Supplemental Table S10). We then extended the co-IP test and used B2-tagged constructs, i.e. giving proteins with a non-native and non-consensus C-terminus (see above), to test 38 putative interactions. Of these, we could test 31 interactions (15 from the cDNA screen, 9 from AD-ORFeome screen, and 7 from both. 27 interactions (87\%) detected by $\mathrm{Y} 2 \mathrm{H}$ were reproduced in these tests, including $12 / 15$ cases where the $\mathrm{Y} 2 \mathrm{H}$ interaction had originally been found only in the cDNA screen using constructs with a native $\mathrm{C}$-terminus (see Additional file 6, Additional file 5: Supplemental Table S11). We thus confirmed many interactions between PDZ domains and proteins with a non-native and/or non-consensus C-terminus. To investigate further the possibility that these PDZ domains were interacting with an internal sequence in the partner protein, we returned to a set of high-confidence interactions found in the cDNA screen. For 59 proteins (corresponding to 74 interactions) that did not possess a canonical consensus $\mathrm{C}$-terminal, we cloned derivatives corresponding to the entire protein less the 3 last residues, or when possible the experimentally defined minimal interacting region (MIR), also without the $3 \mathrm{C}$ terminal residues. This was to ensure that observed interaction did not depend on the native $\mathrm{C}$-terminal residues. Using co-IP, we found that 52/65 interactions (80\%) successfully tested could be reproduced in the coIP system even in the majority of cases when removal of the terminal residues did not create a new consensus binding site (Figure 4, Additional file 7, Additional file 5: Supplemental Tables S12 and S13). We are therefore confident that the dataset that we provide will be a useful source of information to direct studies of PDZdomain signaling pathways.

\section{A $\mathrm{Y} 2 \mathrm{H}$ array as a tool to probe PDZ domain binding}

Many true interactions protein-protein interactions are missed in $\mathrm{Y} 2 \mathrm{H}$ library screens. This high rate of false negatives can be partially alleviated by performing directed $\mathrm{Y} 2 \mathrm{H}$ assays [25-27]. We therefore decided to construct a $\mathrm{Y} 2 \mathrm{H}$ interaction array that would allow candidate proteins to be screened for their binding capacity to the comprehensive set of PDZ domains. For this, we took a collection of yeast strain each expressing a single PDZ-domain from an $\mathrm{Y} 2 \mathrm{H}$ AD-vector, and spotted them in a standard $8 \times 12$ format on a solid agar support. The individual domains on the array can be probed by introducing into each strain a vector allowing the expression of a protein of interest, using a 
A

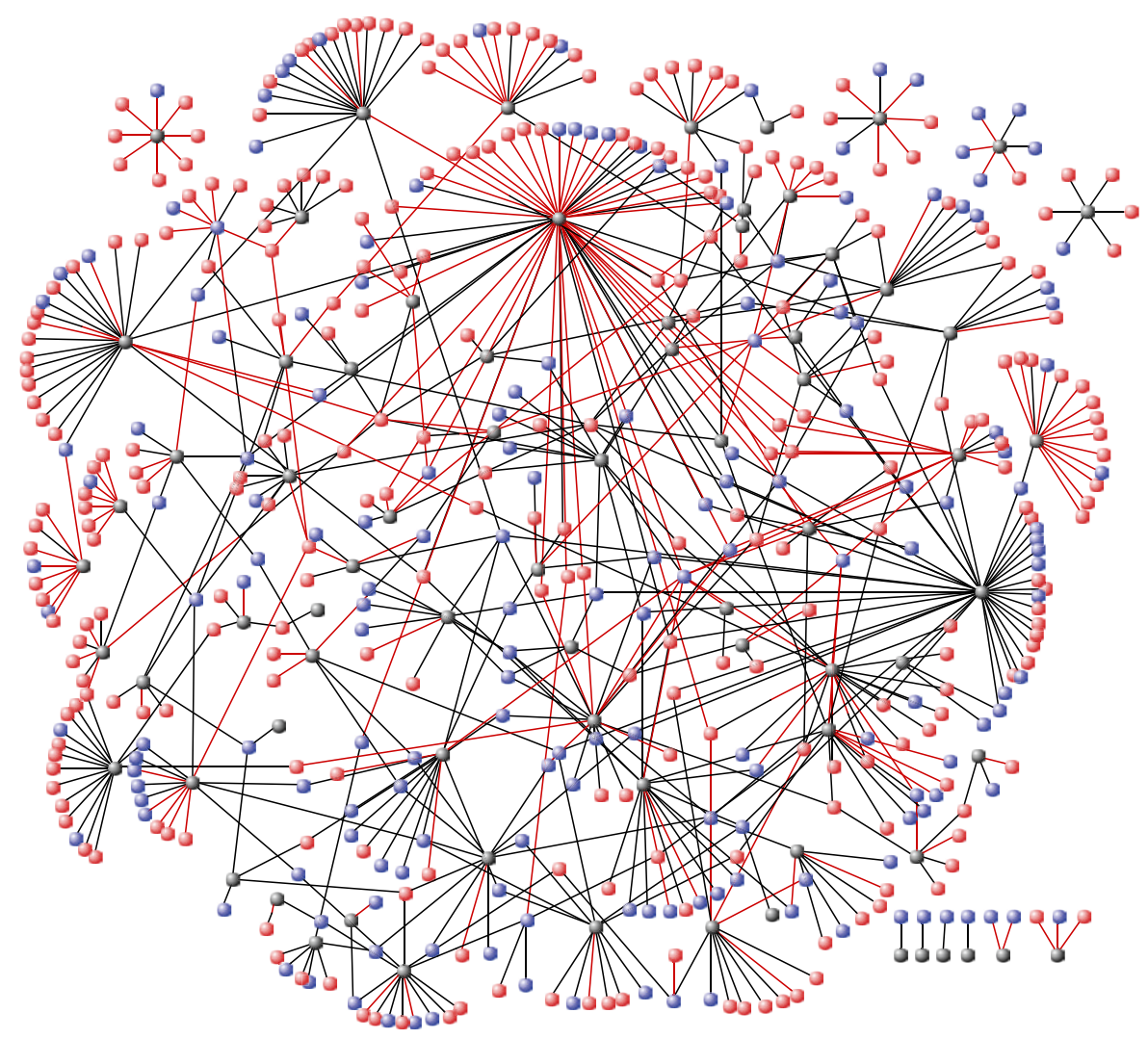

B

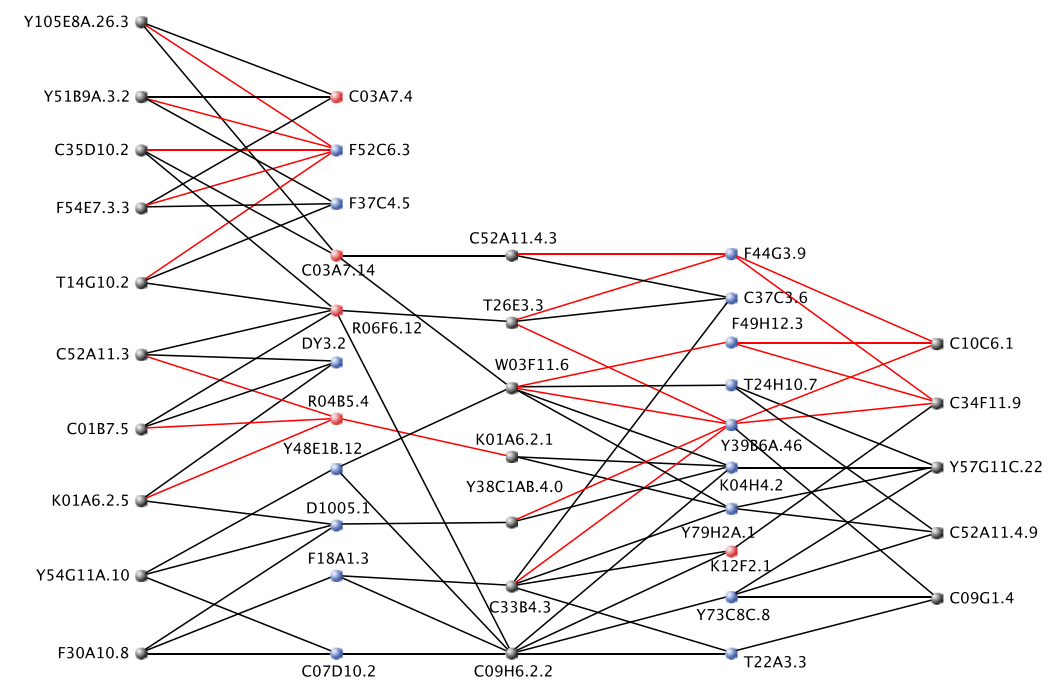

Figure 1 Yeast two-hybrid interactome maps of PDZ domain interactions. Grey nodes represent individual PDZ domains, blue nodes represent interacting proteins having a C-terminal consensus sequence (as defined in Additional file 1) and red nodes represent interacting proteins that do not have a C-terminal consensus sequence. Red edges represent interactions identified in the AD-ORFeome yeast two-hybrid screen and black edges represent interactions identified in the AD-wrmcDNA yeast two-hybrid screen. (A) Global representation of the 674 interactions involving 469 proteins and 78 PDZ domains. (B) Promiscuity: representation of interactions involving selected target proteins with multiple PDZ domains. For multiple PDZ domains in the same protein, a ".n" extension numbered from the ATG was added to the ID of the PDZ containing protein. Some interactions have been omitted for the sake of clarity. Graphs were designed using VisANT [53]. 


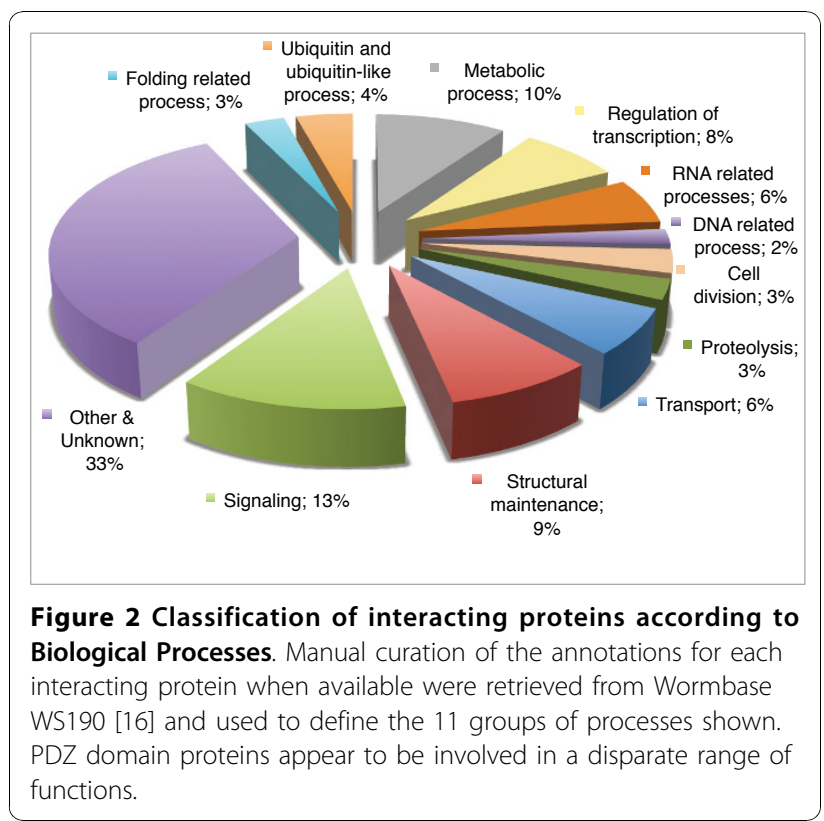

standard $\mathrm{Y} 2 \mathrm{H}$ approach. To test the utility of the resource, we first conducted parallel matings with a yeast strain expressing NRX-1, the C. elegans ortholog of vertebrate neurexin, which plays a critical role in synaptic development (Figure 5). Consistent with the results of our Y2H screen that had identified NRX-1 as an interactor of SYD-1, we found SYD-1 as a partner for NRX-1 using the array. SYD-1 is also a regulator of synaptogenesis. We also identified an additional 4 binding partners, including the single PDZ domain containing STN-2, a gamma syntrophin, and MPZ-1 that can be found at synapses. MPZ-1 has 10 PDZ domains and we detected an interaction only with the 9th domain. We also screened the array with LET-23 and identified 4 proteins, including its known partner LIN-7 [28] (data not shown). On the other hand, when we screened the array with PAC-1, we found PAR-6, which has been demonstrated to be its physiological functional partner [29] (data not shown). Similarly, the sole interactor identified for PRY-1, a negative regulator of Wnt signaling, was MIG-5, one of three C. elegans Dishevelled homologs that functions in both canonical and noncanonical Wnt signaling pathways (Figure 5). Neither of the other 2 Dishevelled homologs were detected as interacting with PRY-1, consistent with previous studies [30].

\section{Discussion}

We constructed a comprehensive, proteome-wide interaction map for all the PDZ domains from C. elegans. Importantly, for a substantial proportion of the interactions, we were able to obtain independent biochemical confirmation. The interactions we characterized covered
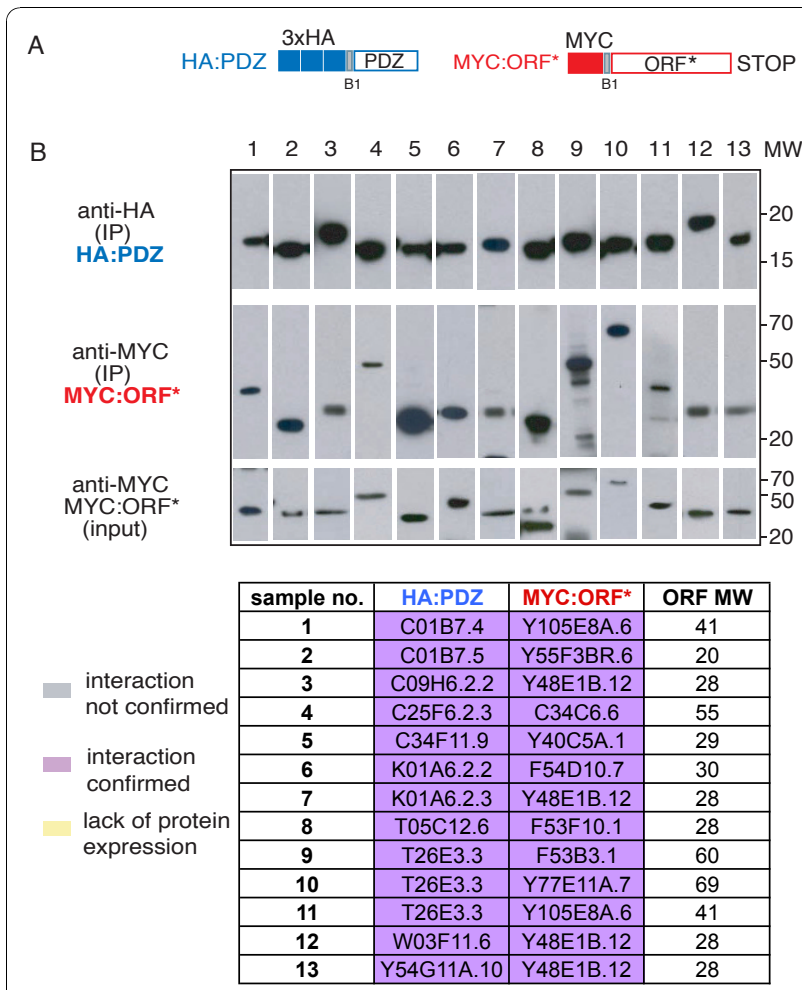

Figure 3 Co-IP verification of yeast two hybrid interacting pairs identified in AD-wrmcDNA screen. (A) Schematic representation of PDZ domains carrying N-terminal 3XHA epitope tag and of their full-length interaction partner terminated with a stop codon (ORF*) carrying N-terminal MYC epitope. Each corresponding pair of constructs to be tested was co-expressed in 293T cells and cellular lysates were subjected to precipitation with anti-HA Sepharose. (B) Presence of interacting protein upon precipitation was revealed by western blotting using anti-MYC serum. For each IP performed three panels are presented. Upper panel: IP reaction probed upon resolution on SDS-PAGE and blotting with anti-HA antibody detecting HA-PDZ domain; Middle panel: the same IP reaction probed with anti-MYC serum detecting ORF (MYC:ORF*); lower panel: detection of expression of each ORF by probing total crude cellular extracts (input) with anti-MYC serum. Table summarizes the interaction pairs tested.

a broad range of putative biological functions, reflecting the ubiquitous involvement of PDZ-domain proteins in cellular physiology. Although a number of PDZ-domain proteins have been functionally characterized in great details, in very few cases has a role for an individual PDZ-domain been identified. We did find a small group of interactions involving PDZ-domains protein for which there was prior experimental evidence, such as those involving LET-23 and LIN-7, and PAR-3 and PKC-3 [28,31]. Further, we were able to provide a molecular basis for certain previously characterized genetic interactions (e.g. between the polarity gene par- 6 and the RhoGAP pac-1 [29]). There were many additional interactions that could merit directed study, such as that 


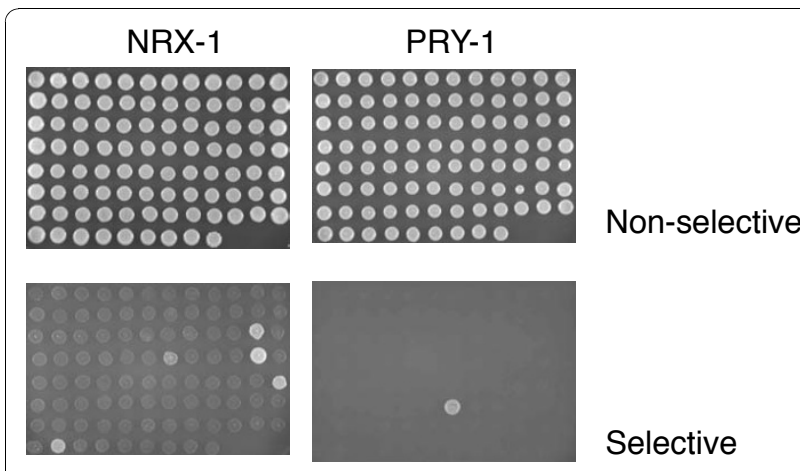

Figure $5 \mathrm{Y} 2 \mathrm{H}$ array for detecting interactions with PDZ domains. An array of yeast strains each expressing one of the $93 \mathrm{C}$. elegans PDZ domains, together with a marker permitting growth on medium lacking leucine, and containing a second vector allowing growth on medium lacking tryptophan as well as expressing NRX-1 (left-hand panels) and PRY-1 (right-hand panels), spotted onto solid agar medium. The upper row shows that all strains grow on medium lacking leucine and tryptophan indicating that all strains contain both prey and bait vectors. In the lower row, on fully selective medium, lacking leucine, tryptophan and histidine, growth only occurs when there is an interaction between a PDZ domain and the protein of interest.

between PTEN/DAF-18 and Dishevelled/DSH-1, two proteins that function respectively in the PTEN/AKT and WNT pathway, and the multipartite interaction between LIN-7 and CSC-1, and LIN-10 with CSC-1 and ICP-1. The LIN-2/LIN-7/LIN-10 complex is known for its role in basolateral targeting of the LET-23 receptor. CSC-1 and ICP-1 are orthologs of Borealin and Incenp two components of the vertebrate chromosomal passenger complex (CPC). We confirmed CSC-1's interactions and the interactions between the two PDZ domains of LIN-10 with ICP-1 using a biochemical approach (JP, unpublished results). This raises the possibility of an unsuspected functional link between these two protein complexes, and is a good example of the hypotheses that can be generated through global analyses.

As a last example, both via our global screen and using the PDZ-domain array, we detected an interaction between MIG-5 and PRY-1. Previous studies had mapped the interaction between PRY-1 and MIG-5 to the N-terminal half of MIG-5 [30], which does contain the protein's single PDZ domain. The C-terminus of PRY-1 (IAAELR) does not contain a consensus PDZ-binding motif. This therefore represents a clear example of a functionally validated protein-protein interaction that we have shown to involve a non-canonical PDZ domain interaction. Indeed, more than half of the interactions did not involve the previously defined PDZ-domain binding C-terminal motifs. By aligning and analyzing our set of PDZ-interacting proteins, we were unable to identify a clear internal motif that could be uniquely responsible for PDZ domain binding. Nevertheless, this global study clearly indicates that non-consensus binding is a much more frequent phenomenon than previously suspected. Extensive future functional studies will be needed to validate all the individual internal PDZ domains interactions described here, but it is important to note that in certain isolated cases, this unconventional mode of binding has been demonstrated [32-42].

It is clear that global $\mathrm{Y} 2 \mathrm{H}$ screens only reveal a fraction of potential protein-protein interactions [43]. Among other factors, this is due to cDNA representation in non-normalized libraries. This was one motivation for generating an array that allows direct $\mathrm{Y} 2 \mathrm{H}$ assay of any protein or peptide of interest against a complete set of PDZ domains. Coupled with the collection of PDZ domain sequences in the Gateway entry vector, allowing facile transfer to vectors for RNAi, or protein expression, the array, which is available as a community resource, will allow comprehensive functional analyses of all PDZ domains in C. elegans.

\section{Conclusions}

By conducting a comprehensive, domain-centered interactome study, we have clearly illustrated at the genome scale the degree of promiscuity and discrimination that governs interactions between individual PDZ domains and their protein partners. This approach also revealed that PDZ domains frequently interact in a non-canonical fashion. This broadens our understanding of PDZ domains and should guide future functional studies.

\section{Methods}

\section{PDZ domain identifications in C. elegans proteome}

Domain boundaries where obtained by cross searching Wormbase WS150 [44] and SMART version 4.0 (genomic mode) (http://smart.embl-heidelberg.de) [45]. Each domain was extended on each side with a 10 amino acid tail from the original protein to ensure the integrity of the structure of the domain. In some cases size of these tails had to be slightly modified according to the position of the PDZ in protein (extreme end or start) or to ensure a correct amplification.

\section{PDZ domain cloning}

Primers, containing Gateway B1 and B2 recombination tails, were designed using the OSP program as described $[46,47]$ including a stop codon before the B2 tail (see Additional file 2: Supplemental Table S1). DNA fragments encoding each PDZ domain where amplified by polymerase chain reaction (Platinum HIFI polymerase, Invitrogen) and cloned into pDONR201 Entry vector using the Gateway recombinational cloning system as described $[17,18]$. PDZ Entry clones were sequence verified using P201DONRF primer 5'-TCGCGTTAACGCTAGCATGGATCTC and then used in a Gateway LR 
recombination reaction to transfer the DNA coding for the PDZ domain into the yeast expression vector pPC97-Dest as described [18].

Transformation of pDB-ORFs into yeast cells and removal of auto-activators

DB-ORF plasmids were transformed into yeast strain MaV203 using standard transformation protocols [48]. Auto-activators were identified by testing the activation of GAL1::HIS3 on minimal medium lacking leucine and histidine but containing $20 \mathrm{mM}$ 3-amino-1,2,4-triazole (3-AT) in the absence of any AD-containing vector.

\section{Identification of interacting protein pairs}

Bait strains containing a single pDB-PDZ were individually transformed with the C. elegans AD-wrmcDNA and AD-ORFeome1.0 libraries [19] as described [48]. A minimum of $1 \times 10^{6}$ colonies were screened for each bait strain tested with the AD-wrmcDNA library and a minimum of $1.5 \times 10^{5}$ colonies were screened for each bait strain tested with the AD-ORFeome library. After 4 to 5 days at $30^{\circ} \mathrm{C}$, single 3 -AT resistant colonies were picked on synthetic complete medium lacking leucine, tryptophan, and histidine and containing $20 \mathrm{mM} 3-\mathrm{AT}$ (SC, Leu-, Trp-, His-, $20 \mathrm{mM} 3-\mathrm{AT}$ ) and then rearrayed on fresh SC, Leu-, Trp-, His-, 20 mM 3-AT plates.

\section{Phenotypic assays}

Colonies able to grow on SC, Leu-, Trp-, His-, $20 \mathrm{mM}$ 3-AT plates were tested for expression of three $\mathrm{Y} 2 \mathrm{H}$ reporter genes (GAL1::HIS3, GAL1::lacZ, and SPAL10:: $U R A 3$, as described [48].

\section{ORF insert sequencing}

To prepare DNA for PCR, yeast colonies were re-suspended in $15 \mu \mathrm{l}$ lysis buffer (50 units zymolase in $0.1 \mathrm{M}$ $\mathrm{Na}$-Phosphate buffer $\mathrm{pH}$ 7.4) using toothpicks, and lysed by incubating for $10 \mathrm{~min}$. at $37^{\circ} \mathrm{C}$ and $10 \mathrm{~min}$. at $95^{\circ} \mathrm{C}$. For each PCR, $0.3 \mu \mathrm{l}$ of lysis mix was used. AD inserts were amplified using primers 5'-CGCGTTTGGAATCACTACAGGG and 5'-GGAGACTTGACCAAACCTCTGGCG (AD and TERM respectively). DB inserts were amplified using primers 5'-GGCTTCAGTGGAGACTGATATGC CTC (DB) and TERM. PCR products were sequenced using the $\mathrm{AD}$ or $\mathrm{DB}$ primers.

\section{Sequence trace analysis}

Colonies showing an activation of at least two of the three $\mathrm{Y} 2 \mathrm{H}$ reporter genes were PCR amplified, as described above. PCR products showing a single band on ethidium bromide gel were sent for sequencing. The quality of the sequence obtained was determined as described [14] by moving a sliding window of 10 base pairs along the sequence to define the portion that has an average PHRED score of 20 or higher $[49,50]$. Sequences for which less than $15 \%$ of their length met this criterion were discarded. A nucleotide BLAST [51] search was performed against Wormpep150 [44] to determine the identity of the clone. Finally, the reading frame was obtained by local alignment of the 3' end of the Gal4 AD encoding sequence with the 5 ' end of the prey encoding sequence. A translation according to this reading frame was used to perform a protein BLAST search against Wormpep150. If the nucleotide and protein BLAST agreed, the prey encoding sequence was considered "In Frame", otherwise it was designated as "Out of Frame" and discarded.

\section{Retesting}

Gap repair was used to retest all $\mathrm{Y} 2 \mathrm{H}$ interactions as described [48]. When an interaction failed to be re-confirmed it was discarded from the dataset.

\section{Construction and screening of a comprehensive PDZ domain $\mathrm{Y} 2 \mathrm{H}$ array}

All PDZ domains were transferred into AD vector (pACT2) by Gateway recombinational cloning and transfected into the haploid Y187 yeast strain (MAT $\alpha$, ura3-

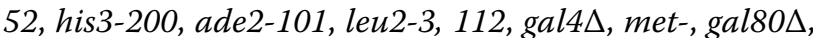
MEL1, URA3::GAL1UAS -GAL1TATA-lacZ). Individual ORFs of proteins of interest were cloned into $D B$ vector (pGBT9) by Gateway recombinational cloning and the resulting constructs transformed into haploid AH109 yeast strain (MATa, trp1-901, leu2-3, 112, ura3-52, his3200, gal4A, gal80A, LYS2::GAL1UAS-GAL1TATA-HIS3, GAL2UAS-GAL2TATA-ADE2, URA3::MEL1UAS-MEL1TATA-lacZ, MEL1). Interactions between each PDZ and a given ORF was tested through mating of the two yeast strains. Phenotypic testing evaluated growth of diploid cells on selective medium (Leu-, Trp-, His-, 2 mM 3-AT), which is dependent in part upon the expression of the GAL1::HIS3 selective marker gene.

\section{Co-IP verification of $\mathrm{Y} 2 \mathrm{H}$ interacting pairs}

To test interactions identified in the AD-wrmcDNA library $\mathrm{Y} 2 \mathrm{H}$ screen using co-IP (Figure 3, Additional file 5: Supplemental Table S10), the full length ORF coding for the target protein identified was amplified from the AD-wrmcDNA library, Gateway cloned into the pDONR201 Entry vector and transferred using the LR reaction into the expression vector $\mathrm{pDEST}-\mathrm{CMV}-\mathrm{MYC}$ which contains a MYC tag upstream of the B1 recombination site. For each fragment the endogenous Stop codon was preserved before the $\mathrm{B} 2$ recombinational tail (the endogenous $\mathrm{C}$-terminus of the corresponding protein fragment was preserved).

To test interactions identified in the AD-wrmcDNA or AD-ORFeome screens, or both (see Additional file 6 
Additional file 5: Supplemental Table S11), but using a B2-tailed construct for each PDZ-domain interacting protein, clones corresponding to the full length protein in the pDONR201 entry vector were retrieved from the C. elegans ORFeome collection [19]. Because of the nature of the constructs used in the ORFeome, LR-transfer of the ORF into the pDEST-CMV-MYC expression vector produced a protein ending with the $\mathrm{C}$-terminal amino-acid sequence PAFLYKVVIIHSSMHLEGPIL (B2 encoding tail +13 aa on pDEST-CMV-MYC before Stop codon).

Internal interactions (Figure 4, Additional file 7, Additional file 5: Supplemental Table S12 and S13) were tested by co-IP using 74 interactions for which the pair of PDZ/interacting proteins was found multiple times through the screening process of the AD-wrmcDNA library but that had no C-terminal consensus PDZ binding motif. These interactions corresponded to 59 different interacting proteins. To ensure a maximum reproducibility with the $\mathrm{Y} 2 \mathrm{H}$ interactions, sequence data from the $\mathrm{Y} 2 \mathrm{H}$ screen was used to define the smallest cDNA fragment identified among all clones obtained for each interaction in the $\mathrm{Y} 2 \mathrm{H}$ screen (designated as the minimal interacting region, or MIR). For each fragment the codons encoding for the last three amino acid were removed from the primers and replaced by a Stop codon, giving rise after PCR amplification, Gateway cloning into the pDONR201 Entry vector and LR-transfer into the pDEST-CMV-MYC expression vector, to a cloned fragment encoding a protein lacking the last three amino acids. This was done to ensure that proteins could not interact by their native $\mathrm{C}$-terminus, so that a positive result would provide support for an internal mode of interaction. When a PDZ-interacting protein was present in multiple pairs of interactions the smallest cDNA fragment of all pairs was used to test all interactions.

For all Co-IP experiments in this study, DNA encoding each PDZ domains tested was transferred from the pDONR201 Entry vector to the pDEST-CMV-3xHA expression vector containing the $3 \times \mathrm{HA}$ sequence upstream of the $\mathrm{B} 1$ recombination site.

Plasmids pDEST-CMV-3xHA and pDEST-CMV-MYC expressing their fusion proteins from the CMV promoters were transfected into 293T cells using Fugen 6 transfection reagent according to the manufacturers instructions (Roche). Cells were cultured for 48 hours in DMEM medium, and lysed in $0.1 \%$ NP-40 buffer (50 mM Tris- $\mathrm{HCl}, \mathrm{pH}$ 7.5, $150 \mathrm{mM} \mathrm{NaCl}, 1 \mathrm{mM}$ EDTA and complete protease inhibitors and phosphatase inhibitors (Thermo Scientific)). Lysates were cleared by centrifugation at $14,000 \times \mathrm{g}$ and subjected to co-immunoprecipitations of protein complexes using anti-HA (clone12CA5) sepharose beads. Purified complexes and control lysate $(10 \mu \mathrm{g}$ of total protein) samples were separated on $\mathrm{Nu}-\mathrm{PAGE}$ Bis-Tris 4-12\% gels (Invitrogen), and MYC and HA tagged proteins were detected using standard immunoblotting techniques. Antibodies used were mouse monoclonal anti-MYC (clone 9E10, Sigma) and monoclonal anti-HA (clone HA.11, Covance).

\section{Database searches}

The Textpresso database [52] was used to search for interactions were both bait and prey proteins had public alphanumeric gene names. GO terms attributes were retrieved from Wormbase. Note that in C. elegans most attributes are currently inferred from electronic annotation (IEA).

\section{Additional material}

Additional file 1: Definition of consensus classes. Additional file 1 is a table describing the consensus classes used in this study. We defined for this study three extended consensus classes encompassing the different definitions available so far, so as to have the broadest definition of classes $[1,10]$

Additional file 2: PDZ domains cloning and annotations. Additional file 2 contains two tables (S1 and S2) listing for each PDZ domain identified, respectively the primer sequences and the Gene Ontology annotations. Supplemental Table S1: List of primers used to clone PDZ domains. Proteins names and IDs are given according to Wormbase WS150 [44]. For multiple PDZ domains in the same protein, a ".n" extension was added to the ID of the PDZ containing protein. This extension was numbered from the ATG (eg: F54E7.3.1 is the ID for the first PDZ domain of F54E7.3). When only one PDZ was present, protein ID was kept as such. Coordinates on PDZ domain containing proteins correspond to the splice form specified in the third column (PDZ domain containing protein ID). Each primer contains the B1 and B2 Gateway recombination cloning tail. Supplemental Table S2: Gene Ontology annotation based on experimental data for PDZ domains proteins. Gene ontology annotations were retrieved from Wormbase WS190 [16]. Experimental Evidence Codes: EXP: Inferred from Experiment, IDA: Inferred from Direct Assay, IPI: Inferred from Physical Interaction, IMP: Inferred from Mutant Phenotype, IGI: Inferred from Genetic Interaction, IEP: Inferred from Expression Pattern

Additional file 3: PDZome network. Additional file 3 contains four tables (S3 to S6) listing the PDZome network interacting pairs and giving statistical analysis of the interactions. Supplemental Table S3: Two hybrid screen results. Gene names and ID are given according to Wormbase WS150[44]. Number of hits refers to the number of independent colonies identified and phenotypically tested for each interacting partner. Number of splice-forms identified or predicted in Wormbase WS150 and last 6 amino acids of each splice-form are given in cases were several spliceforms are identified or predicted. When sequencing from the N-terminus did not span the entire fragment, and thus the C-terminus was not experimentally confirmed, if any of the predicted splice-form had a Cterminal consensus motif, to be conservative, a consensus class was attributed. Consensus class type: [ST]X[YFWCMVILA] $=1$; [YFWCMVILA]X $[$ YFWCMVILA] $=2 ;$ [DE]X[YFWCMVILA] $=3$ (X: any amino acid).

Supplemental Table S4: Promiscuity and specificity of PDZ interactome network. Number of independent interacting proteins per PDZ domain, and number of PDZ domains interacting with each protein are given for AD-wrmcDNA and AD-ORFeome libraries two-hybrid screens.

Supplemental Table S5: Promiscuity and specificity of PDZ interactome network: mean and median for number of interacting proteins per PDZ or vice versa. Supplemental Table S6: Number of interacting proteins per consensus class in network. Single hits: interacting proteins for which only one clone was identified in two-hybrid screens. Multiple hits: interacting proteins for which more than one clone was identified in two-hybrid screens. 


\section{Additional file 4: Functional annotation of interacting proteins} Additional file 4 contains three tables (S7 to S9) listing the functional annotations of the PDZ domain interacting proteins. Supplemental Table S7: Concise description, GO terms and KOG (EuKaryotic Orthologous Groups), for each interacting protein when available, retrieved from Wormbase WS 190 [16]; note that most attributes are inferred from electronic annotation. Experimental Evidence Codes: EXP: Inferred from Experiment, IDA: Inferred from Direct Assay, IPI: Inferred from Physical Interaction, IMP: Inferred from Mutant Phenotype, IGI: Inferred from Genetic Interaction, IEP: Inferred from Expression Pattern. Computational Analysis Evidence Codes: ISS: Inferred from Sequence or Structural Similarity, ISO: Inferred from Sequence Orthology, ISA: Inferred from Sequence Alignment, ISM: Inferred from Sequence Model, IGC: Inferred from Genomic Context, RCA: inferred from Reviewed Computational Analysis. Author Statement Evidence Codes: TAS: Traceable Author Statement, NAS: Non-traceable Author Statement. Curator Statement Evidence Codes: IC: Inferred by Curator, ND: No biological Data available. Automatically-assigned Evidence Codes: IEA: Inferred from Electronic Annotation. Supplemental Table S8: Classification of interacting proteins according to Cellular Components terms: integral to membrane, nucleus and other\&unknown. Supplemental Table S9: Classification of interacting proteins according to Biological Processes: manual curation of annotations retrieved from Wormbase WS190 were used to define the 11 groups of processes shown in Figure 2.

Additional file 5: Verification of two-hybrid interacting pairs by coimmunoprecipitation. Additional file 5 contains four tables (S10 to S13) listing interacting proteins pairs tested and results. Supplemental Table S10 lists tested pairs involving PDZ domains and their respective twohybrid identified interacting proteins possessing a free C-terminus. Supplemental Table S11 lists tested pairs involving PDZ domains and their respective two-hybrid identified interacting proteins using a B2 tailed construct. Supplemental Table S12 lists the 59 protein fragments that did not possess a C-terminal binding motif tested by $\mathrm{CO}_{-}$ immunoprecipitation in a C-terminally truncated form against their respective two hybrid interacting PDZs. Sequences where there was creation of a new C-terminal consensus sites after truncation are shown. Supplemental Table S13 lists the primers pairs used to amplify and clone truncated interacting protein fragments used in the coimmunoprecipitation experiment.

Additional file 6: Co-IP verification of yeast two hybrid interacting pairs identified in AD-wrmcDNA and AD-ORFeome screens using a B2 tailed construct. Additional file 6 is a figure showing the Co-IP verification of yeast two hybrid interacting pairs identified in $A D$ wrmcDNA and AD-ORFeome screens using a B2 tailed construct. (A) Schematic representation of PDZ domains carrying $\mathrm{N}$-terminal $3 \mathrm{XHA}$ epitope tag and of their interacting protein, ending with the B2 tail, carrying N-terminal MYC epitope. (B) Each pair of constructs to be tested was co-expressed in 293T cells and co-IP was performed using cellular lysates subjected to precipitation with anti-HA sepharose. Presence of interacting protein upon precipitation was revealed by western blotting using anti-MYC serum. For each IP performed three panels are presented. Upper panel: IP reaction probed upon resolution on SDS-PAGE and blotting with anti-HA antibody detecting HA-PDZ domain; Middle panel: the same IP reaction probed with anti-MYC serum detecting ORF (MYC: ORF); lower panel: detection of expression of each ORF by probing total crude cellular extracts (input) with anti-MYC serum. Table summarizes the interaction pairs tested and color code is used to indicate the outcome (purple: interaction tested positive, grey: no interaction and yellow: inconclusive as one or both partners are not expressed). (C) Each ORF used in above co-IP experiment was also subjected to co-transfection and co-immunoprecipitation with empty PDEST-CMV-3xHA vector to serve as a negative control for the binding assay. Detection and analysis were performed as above.

Additional file 7: Negative controls of immunoprecipitations shown in Figure 4. Additional file 7 is a figure showing the test for unspecific binding of non-consensus C-terminally truncated proteins (MYC: MIRdCter) to irrelevant HA epitoped peptide in co-immunoprecipitation reaction corresponding to negative control of the experiment described in Figure 4. Each MYC:MIRdCter construct was co-expressed in 293T cells together with empty pDEST-CMV-3xHA vector and co-IPed using anti-HA sepharose beads. Binding of given protein upon precipitation was revealed by western blotting using anti-MYC serum. For each IP performed three panels are presented. Upper panel: IP reaction probed after resolution on SDS-PAGE and blotting with anti-HA antibody. Middle panel: the same IP reaction probed with anti-MYC serum detecting the truncated protein fragments (MYC:MIRdCter). Lower panel: detection of expression of each truncated protein fragment by probing total crude cellular extracts (input) with anti-MYC serum.

\section{Acknowledgements}

We thank Jonathan Ewbank. This work was supported by an institutional grant from INSERM, the INSERM-AVENIR program and Association pour la Recherche sur le Cancer. N.L. was funded by INSERM-Region PACA and Association pour la Recherche sur le Cancer PhD fellowship. S.B. was a recipient of INSERM Poste Vert fellowship.

\section{Author details}

'Inserm, U891, CRCM, Marseille, F-13009, France; Institut Paoli-Calmettes, Marseille, F-13009, France; Univ Méditerranée, F-13007, Marseille, France. ${ }^{2}$ Institute of Integrative and Comparative Biology, Faculty of Biological Sciences, The University of Leeds, Leeds, LS2 9JT, UK. ${ }^{3}$ CIML, Parc Scientifique et Technologique de Luminy Case 906 - 13288 Marseille - Cedex 9, France.

\section{Authors' contributions}

$\mathrm{NL}, J \mathrm{P}, \mathrm{SB}$ and SO carried out the experiments. NL performed the bioinformatic analysis. NL, JP and JR analyzed the data. JP, JPB and JR conceived the study. JR wrote the manuscript. All authors have read and approved the final manuscript.

Received: 15 September 2010 Accepted: 26 November 2010 Published: 26 November 2010

\section{References}

1. Nourry C, Grant SG, Borg JP: PDZ domain proteins: plug and play! SCi STKE 2003, 2003(179):RE7.

2. Roh MH, Margolis B: Composition and function of PDZ protein complexes during cell polarization. Am J Physiol Renal Physiol 2003, 285(3): F377-387.

3. Feng W, Zhang M: Organization and dynamics of PDZ-domain-related supramodules in the postsynaptic density. Nat Rev Neurosci 2009, 10(2):87-99.

4. Saras J, Heldin CH: PDZ domains bind carboxy-terminal sequences of target proteins. Trends Biochem Sci 1996, 21(12):455-458.

5. Kurakin A, Swistowski A, Wu SC, Bredesen DE: The PDZ domain as a complex adaptive system. PLOS ONE 2007, 2(9):e953.

6. Song E, Gao S, Tian R, Ma S, Huang H, Guo J, Li Y, Zhang L, Gao Y: A high efficiency strategy for binding property characterization of peptidebinding domains. Mol Cell Proteomics 2006, 5(8):1368-1381.

7. Giallourakis C, Cao Z, Green T, Wachtel H, Xie X, Lopez-lllasaca M, Daly M, Rioux J, Xavier R: A molecular-properties-based approach to understanding PDZ domain proteins and PDZ ligands. Genome Res 2006, 16(8):1056-1072.

8. He J, Bellini M, Inuzuka H, Xu J, Xiong Y, Yang X, Castleberry AM, Hall RA: Proteomic analysis of beta1-adrenergic receptor interactions with PDZ scaffold proteins. J Biol Chem 2006, 281(5):2820-2827.

9. Stiffler MA, Chen JR, Grantcharova VP, Lei Y, Fuchs D, Allen JE, Zaslavskaia $L A$, MacBeath G: PDZ domain binding selectivity is optimized across the mouse proteome. Science 2007, 317(5836):364-369.

10. Tonikian R, Zhang Y, Sazinsky SL, Currell B, Yeh JH, Reva B, Held HA, Appleton BA, Evangelista M, Wu Y, et al: A specificity map for the PDZ domain family. PLOS Biol 2008, 6(9):e239.

11. Harris BZ, Lim WA: Mechanism and role of PDZ domains in signaling complex assembly. J Cell Sci 2001, 114(Pt 18):3219-3231.

12. Songyang Z, Fanning AS, Fu C, Xu J, Marfatia SM, Chishti AH, Crompton A, Chan AC, Anderson JM, Cantley LC: Recognition of unique carboxylterminal motifs by distinct PDZ domains. Science 1997, 275(5296):73-77.

13. Uniprot: [http://www.uniprot.org]. [http://www.uniprot.org/uniprot/ 075970]. 
14. Li S, Armstrong CM, Bertin N, Ge H, Milstein S, Boxem M, Vidalain PO, Han JD, Chesneau A, Hao T, et al: A map of the interactome network of the metazoan C. elegans. Science 2004, 303(5657):540-543.

15. Simonis N, Rual JF, Carvunis AR, Tasan M, Lemmens I, Hirozane-Kishikawa T, Hao T, Sahalie JM, Venkatesan K, Gebreab F, et al: Empirically controlled mapping of the Caenorhabditis elegans protein-protein interactome network. Nat Methods 2009, 6(1):47-54.

16. Wormbase data freeze 190. [http://ws190.wormbase.org/].

17. Hartley JL, Temple GF, Brasch MA: DNA cloning using in vitro site-specific recombination. Genome Res 2000, 10(11):1788-1795.

18. Walhout AJ, Temple GF, Brasch MA, Hartley JL, Lorson MA, van den Heuvel S, Vidal M: GATEWAY recombinational cloning: application to the cloning of large numbers of open reading frames or ORFeomes. Methods Enzymol 2000, 328:575-592.

19. Reboul J, Vaglio P, Rual JF, Lamesch P, Martinez M, Armstrong CM, Li S, Jacotot $L$, Bertin $N$, Janky $R$, et al: $C$. elegans ORFeome version 1.1: experimental verification of the genome annotation and resource for proteome-scale protein expression. Nat Genet 2003, 34(1):35-41.

20. Basdevant N, Weinstein $H$, Ceruso M: Thermodynamic basis for promiscuity and selectivity in protein-protein interactions: PDZ domains, a case study. J Am Chem Soc 2006, 128(39):12766-12777.

21. Ashburner M, Ball CA, Blake JA, Botstein D, Butler H, Cherry JM, Davis AP, Dolinski K, Dwight SS, Eppig JT, et al: Gene ontology: tool for the unification of biology. The Gene Ontology Consortium. Nat Genet 2000, 25(1):25-29.

22. Craven SE, Bredt DS: PDZ proteins organize synaptic signaling pathways. Cell 1998, 93(4):495-498.

23. Kim SK: Polarized signaling: basolateral receptor localization in epithelial cells by PDZ-containing proteins. Curr Opin Cell Biol 1997, 9(6):853-859.

24. Traweger A, Fuchs R, Krizbai IA, Weiger TM, Bauer HC, Bauer H: The tight junction protein ZO-2 localizes to the nucleus and interacts with the heterogeneous nuclear ribonucleoprotein scaffold attachment factor-B. J Biol Chem 2003, 278(4):2692-2700.

25. Walhout AJ, Sordella R, Lu X, Hartley JL, Temple GF, Brasch MA, ThierryMieg N, Vidal M: Protein interaction mapping in C. elegans using proteins involved in vulval development. Science 2000, 287(5450):116-122.

26. Uetz P, Giot L, Cagney G, Mansfield TA, Judson RS, Knight JR, Lockshon D, Narayan $V$, Srinivasan M, Pochart $P$, et al: A comprehensive analysis of protein-protein interactions in Saccharomyces cerevisiae. Nature 2000, 403(6770):623-627.

27. Vermeirssen V, Deplancke B, Barrasa MI, Reece-Hoyes JS, Arda HE, Grove CA, Martinez NJ, Sequerra R, Doucette-Stamm L, Brent MR, et al: Matrix and Steiner-triple-system smart pooling assays for high-performance transcription regulatory network mapping. Nat Methods 2007, 4(8):659-664.

28. Kaech SM, Whitfield CW, Kim SK: The LIN-2/LIN-7/LIN-10 complex mediates basolateral membrane localization of the $\mathrm{C}$. elegans EGF receptor LET-23 in vulval epithelial cells. Cell 1998, 94(6):761-771.

29. Anderson DC, Gill JS, Cinalli RM, Nance J: Polarization of the C. elegans embryo by RhoGAP-mediated exclusion of PAR-6 from cell contacts. Science 2008, 320(5884):1771-1774.

30. Korswagen HC, Coudreuse DY, Betist MC, van de Water S, Zivkovic D, Clevers HC: The Axin-like protein PRY-1 is a negative regulator of a canonical Wnt pathway in C. elegans. Genes Dev 2002, 16(10):1291-1302.

31. Tabuse Y, Izumi Y, Piano F, Kemphues KJ, Miwa J, Ohno S: Atypical protein kinase $C$ cooperates with PAR-3 to establish embryonic polarity in Caenorhabditis elegans. Development 1998, 125(18):3607-3614.

32. Eldstrom J, Doerksen KW, Steele DF, Fedida D: N-terminal PDZ-binding domain in Kv1 potassium channels. FEBS Lett 2002, 531(3):529-537.

33. Im YJ, Lee JH, Park SH, Park SJ, Rho SH, Kang GB, Kim E, Eom SH: Crystal structure of the Shank PDZ-ligand complex reveals a class I PDZ interaction and a novel PDZ-PDZ dimerization. J Biol Chem 2003, 278(48):48099-48104

34. Wong HC, Bourdelas A, Krauss A, Lee HJ, Shao Y, Wu D, Mlodzik M, Shi DL, Zheng J: Direct binding of the PDZ domain of Dishevelled to a conserved internal sequence in the C-terminal region of Frizzled. $\mathrm{Mol}$ Cell 2003, 12(5):1251-1260.

35. London TB, Lee HJ, Shao Y, Zheng J: Interaction between the internal motif KTXXXI of Idax and mDvI PDZ domain. Biochem Biophys Res Commun 2004, 322(1):326-332.
36. Penkert RR, DiVittorio HM, Prehoda KE: Internal recognition through PDZ domain plasticity in the Par-6-Pals1 complex. Nat Struct Mol Biol 2004, 11(11):1122-1127.

37. Ellencrona K, Syed A, Johansson M: Flavivirus NS5 associates with hostcell proteins zonula occludens-1 (ZO-1) and regulating synaptic membrane exocytosis-2 (RIMS2) via an internal PDZ binding mechanism. Biol Chem 2009, 390(4):319-323.

38. Tochio H, Mok YK, Zhang Q, Kan HM, Bredt DS, Zhang M: Formation of nNOS/PSD-95 PDZ dimer requires a preformed beta-finger structure from the nNOS PDZ domain. J Mol Biol 2000, 303(3):359-370.

39. Zhang Y, Appleton BA, Wu P, Wiesmann C, Sidhu SS: Structural and functional analysis of the ligand specificity of the HtrA2/Omi PDZ domain. Protein Sci 2007, 16(8):1738-1750.

40. Lemaire JF, McPherson PS: Binding of Vac14 to neuronal nitric oxide synthase: Characterisation of a new internal PDZ-recognition motif. FEBS Lett 2006, 580(30):6948-6954.

41. Utepbergenov DI, Fanning AS, Anderson JM: Dimerization of the scaffolding protein ZO-1 through the second PDZ domain. J Biol Chem 2006, 281(34):24671-24677

42. Hillier BJ, Christopherson KS, Prehoda KE, Bredt DS, Lim WA: Unexpected modes of PDZ domain scaffolding revealed by structure of nNOSsyntrophin complex. Science 1999, 284(5415):812-815.

43. Huang $\mathrm{H}$, Jedynak BM, Bader JS: Where have all the interactions gone? Estimating the coverage of two-hybrid protein interaction maps. PLOS Comput Biol 2007, 3(11):e214.

44. Wormbase data freeze 150. [http://ws150.wormbase.org/].

45. Schultz J, Copley RR, Doerks T, Ponting CP, Bork P: SMART: a web-based tool for the study of genetically mobile domains. Nucleic Acids Res 2000, 28(1):231-234.

46. Hillier L, Green P: OSP: a computer program for choosing PCR and DNA sequencing primers. PCR Methods App/ 1991, 1(2):124-128.

47. Reboul J, Vaglio P, Tzellas N, Thierry-Mieg N, Moore T, Jackson C, Shin-i T, Kohara Y, Thierry-Mieg D, Thierry-Mieg J, et al: Open-reading-frame sequence tags (OSTs) support the existence of at least 17,300 genes in C. elegans. Nat Genet 2001, 27(3):332-336.

48. Walhout AJ, Vidal M: High-throughput yeast two-hybrid assays for largescale protein interaction mapping. Methods 2001, 24(3):297-306.

49. Ewing B, Green P: Base-calling of automated sequencer traces using phred. II. Error probabilities. Genome Res 1998, 8(3):186-194.

50. Ewing B, Hillier L, Wend MC, Green P: Base-calling of automated sequencer traces using phred. I. Accuracy assessment. Genome Res 1998, 8(3):175-185.

51. Altschul SF, Gish W, Miller W, Myers EW, Lipman DJ: Basic local alignment search tool. J Mol Biol 1990, 215(3):403-410.

52. The textpresso database. [http://textpresso.org].

53. Hu Z, Mellor J, Wu J, DeLisi C: VisANT: an online visualization and analysis tool for biological interaction data. BMC Bioinformatics 2004, 5:17.

doi:10.1186/1471-2164-11-671

Cite this article as: Lenfant et al:: A genome-wide study of PDZ-domain interactions in C. elegans reveals a high frequency of non-canonical binding. BMC Genomics 2010 11:671.

\section{Submit your next manuscript to BioMed Central and take full advantage of:}

- Convenient online submission

- Thorough peer review

- No space constraints or color figure charges

- Immediate publication on acceptance

- Inclusion in PubMed, CAS, Scopus and Google Scholar

- Research which is freely available for redistribution

Submit your manuscript at www.biomedcentral.com/submit
Biomed Central 